\title{
Axial Movements and Length Changes of the Human Lower Esophageal Sphincter During Respiration and Distension-induced Secondary Peristalsis Using Functional Luminal Imaging Probe
}

\author{
Donghua Liao, ${ }^{1}$ Christian Lottrup, ${ }^{2}$ Lotte Fynne, ${ }^{3}$ Barry P McMahon, ${ }^{4}$ Klaus Krogh, ${ }^{3}$ Asbjørn M Drewes, ${ }^{2}$ Jingbo Zhao, ${ }^{1}$ and \\ Hans Gregersen ${ }^{5 *}$ \\ ${ }^{I}$ GIOME Academia, Department of Medicine, Aarhus University, Aarhus, Denmark; ${ }^{2}$ Mech-Sense, Department of Gastroenterology and \\ Hepatology, Aalborg University Hospital, Aalborg, Denmark; ${ }^{3}$ Neurogastroenterology Unit, Department of Hepatology and Gastroenterology, \\ Aarhus University Hospital, Aarhus, Denmark; ${ }^{4}$ TAGG Research Center, Tallaght Hospital and Trinity College, Dublin, Ireland; and ${ }^{5}$ GIOME, \\ Department of Surgery, Chinese University of Hong Kong and Prince of Wales Hospital, Hong Kong
}

\section{Background/Aims}

Efficient transport through the esophago-gastric junction (EGJ) requires synchronized circular and longitudinal muscle contraction of the esophagus including relaxation of the lower esophageal sphincter (LES). However, there is a scarcity of technology for measuring esophagus movements in the longitudinal (axial) direction. The aim of this study is to develop new analytical tools for dynamic evaluation of the length change and axial movement of the human LES based on the functional luminal imaging probe (FLIP) technology and to present normal signatures for the selected parameters.

\section{Methods}

Six healthy volunteers without hiatal hernia were included. Data were analyzed from stepwise LES distensions at 20,30, and $40 \mathrm{~mL}$ bag volumes. The bag pressure and the diameter change were used for motion analysis in the LES. The cyclic bag pressure frequency was used to distinguish dynamic changes of the LES induced by respiration and secondary peristalsis.

\section{Results}

Cyclic fluctuations of the LES were evoked by respiration and isovolumetric distension, with phasic changes of bag pressure, diameter, length, and axial movement of the LES narrow zone. Compared to the respiration-induced LES fluctuations, peristaltic contractions increased the contraction pressure amplitude $(P<0.001)$, shortening $(P<0.001)$, axial movement $(P<0.001)$, and diameter change $(P<0.01)$ of the narrow zone. The length of the narrow zone shortened as function of the pressure increase.

\section{Conclusions}

FLIP can be used for evaluation of dynamic length changes and axial movement of the human LES. The method may shed light on abnormal longitudinal muscle activity in esophageal disorders.

(J Neurogastroenterol Motil 2018;24:255-267)

Key Words

Distension; Esophageal sphincter, lower; Longitudinal; Motion

Received: July 4, 2017 Revised: September 29, 2017 Accepted: December 12, 2017

(.) This is an Open Access article distributed under the terms of the Creative Commons Attribution Non-Commercial License (http://creativecommons. org/licenses/by-nc/4.0) which permits unrestricted non-commercial use, distribution, and reproduction in any medium, provided the original work is properly cited.

*Correspondence: Hans Gregersen, MD, PhD

Department of Surgery, 4/F, Lui Che Woo Clinical Sciences Building, Prince of Wales Hospital, Shatin, Hong Kong Tel: +852-2632-1609, Fax: +852-2635-3487, E-mail: hag@giome.org 


\section{Introduction}

The major function of the lower esophageal sphincter (LES) is to allow food to pass from the esophagus into the stomach by relaxing immediately before and during passage of the food bolus and to prevent reflux of gastric contents into the esophagus by maintaining tonic contraction at rest. LES efficacy requires synchronized circular and longitudinal muscle activity of the esophagus. Lack of coordination between the integrated muscle actions of the esophagus body and LES is associated with symptoms and esophageal disorders such as dysphagia, achalasia, and unexplained chest pain. ${ }^{1-3}$ Consequently, understanding the role of the circular and longitudinal muscle contractile activity in the LES region is mandatory for understanding the function.

In general, current intra-luminal pressure or impedance planimetry recording technologies were developed primarily for studying the circular muscle activities of the esophagus by means of measuring the pressure or cross-sectional area (CSA) change. ${ }^{4-9}$ However, technology is lagging for recording longitudinal muscle activity in the human esophagus. Longitudinal muscle contraction and relaxation cause axial shortening and lengthening of the esophagus. A major problem in studying the longitudinal muscle layer of the esophagus, unlike circular muscle, is the difficulty in measuring its contraction and relaxation. Investigators have used implanted radiopaque markers, ${ }^{10-14}$ strain gauges, ${ }^{3}$ or high frequency intraluminal ultrasound imaging (HFIUS) ${ }^{7,15-19}$ to study longitudinal muscle contraction of the esophagus as well as axial movement of the LES during respiration, ${ }^{20}$ swallowing and during transient LES relaxation (TLESR). ${ }^{10-12,14,21}$ In addition to the upward movement of the LES, studies done by Shi et $\mathrm{al}^{14}$ showed the most distal part of the esophagus (segment between the squamo-columnar junction and $3 \mathrm{~cm}$ proximal to the squamo-columnar junction) had a greater shortening than the proximal segment during both primary and secondary peristalsis, revealing shortening of the LES region during peristaltic contractions. Placement of clips or markers requires endoscopic access and can be technically challenging due to peristalsis and respiratory movement. ${ }^{22}$ HFIUS is limited by the time-consuming image processing and data analysis ${ }^{17}$ and the validity is challenged due to movement of the esophagus relative to the stationary HFIUS catheter. Mittal et $\mathrm{al}^{8}$ have measured proximal movements of the LES high-pressure zone (LES lift) using highresolution manometry (HRM). The LES lift is believed to be a result of esophageal longitudinal muscle contraction. However, HRM only measures LES movement during TLESRs without total loss of tone (incomplete TLESRs) ${ }^{8}$ whereas secondary esophageal peristalsis is not assessed. None of the technologies mentioned above can provide simultaneous recordings of the shortening, axial movement and diameter change of the LES during peristalsis. Such measurements would add another dimension to our understanding of LES physiology by studying the interaction between lower esophageal distension and distension-induced secondary peristalsis.

The functional luminal imaging probe (FLIP) was developed for assessment of luminal size and sphincter distensibility in the gastrointestinal tract. ${ }^{5,6,23-25}$ FLIP measurement of geometric profiles along with pressure measurement provides dynamic evaluation of LES distensibility and efficacy. Recently, a series of clinical FLIP studies have been published on patients suffering from achalasia, ${ }^{26,27}$ esophago-gastric junction (EGJ) outflow obstruction, ${ }^{28}$ and systemic sclerosis. ${ }^{29,30}$ In this study, the LES was distended in healthy volunteers without hiatal hernia using FLIP. Dynamic changes of the LES length, diameters and axial movement induced by respiration and secondary peristaltic contraction were evaluated during the distensions. The aims of this study are (1) to provide an advanced data analysis method for FLIP measurement, and (2) to quantify the dynamic longitudinal and circumferential change of LES during respiration and secondary peristalsis.

\section{Materials and Methods}

\section{Subject Selection}

Subjects included in this study were retrospectively selected from a database of FLIP studies conducted in the EGJ at MechSense, Aalborg University Hospital and the Neurogastroenterology Unit, Aarhus University Hospital, Denmark. Recordings from 6 healthy volunteers (age $53.8 \pm 10.3$ years, body mass index $24.3 \pm$ 1.1) without hiatal hernias as judged by upper endoscopy were selected for analysis. ${ }^{5,29}$ It was a criteria that the whole LES region was visible at all times during the distensions. The protocol and written information was approved by the local Ethical Committee (project ID N-20090008 and M-1-10-72-330-13). Written informed consent was received from all subjects.

\section{Study Protocol}

\section{Functional luminal imaging probe}

FLIP is a $1-\mathrm{m}$-long probe with a $12-\mathrm{cm}-$ long cylindrical bag mounted close to the tip (EF325-N; Crospon Ltd, Galway, Ireland). The bag can be filled with saline and emptied using a pump 
in the equipment. The probe measures the pressure and CSAs of the bag at 16 axial locations $5 \mathrm{~mm}$ apart using electrical impedance measurements inside the bag. ${ }^{6,23}$ The volume, intra-bag pressure, and 16 CSAs were recorded simultaneously at a sampling rate of 10 $\mathrm{Hz}$ for each signal and stored for later analysis.

\section{Distension protocol}

The probe was passed orally through the esophagus into the stomach with the subject sitting upright. After infusion of $10 \mathrm{~mL}$ saline into the bag, it was slowly retracted to the preferred position in the LES. The probe was centered in the sphincter as visualized on the FLIP monitor with the narrowing region in the middle of the bag (Fig. 1A). This means that the proximal part of the bag distends in the lower esophageal body and the distal part of the bag distends in the stomach. The bag was emptied and after a short rest period the subject was ready for measurements. The subjects were asked to hold swallows to avoid primary peristalsis that would make

\section{A}

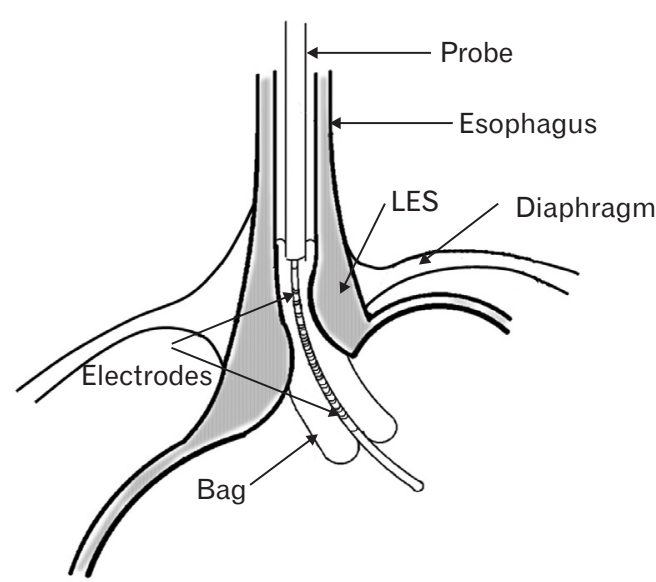

\section{C}

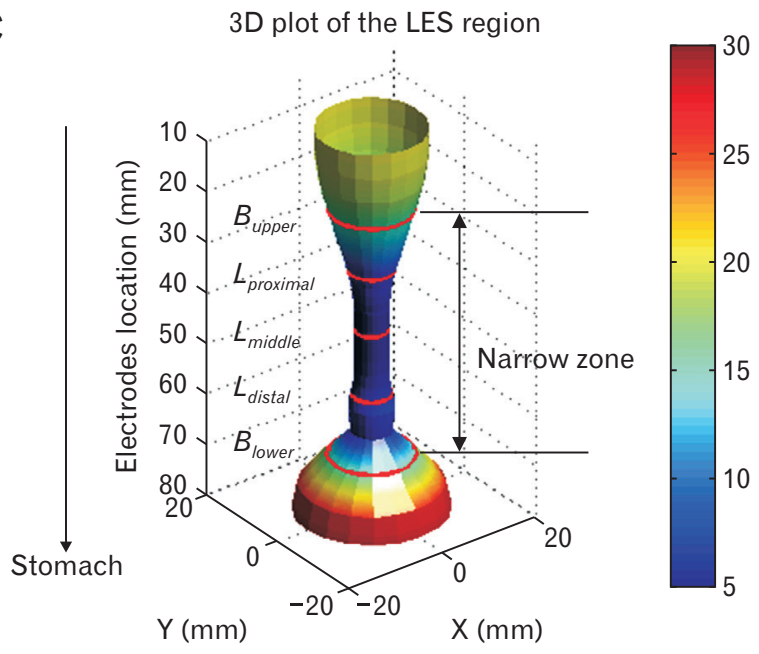

it more difficult to analyze the secondary peristalsis induced by the distension.

The stepwise distension started with the empty bag being filled at a rate of $30 \mathrm{~mL} / \mathrm{min}$ to a maximum bag volume of $50 \mathrm{~mL}$. Intermediate distensions at 20, 30, 40, and $50 \mathrm{~mL}$ were made. Each volume step plateau lasted at least 30 seconds (Fig. 1B). The bag pressure and the spatio-temporal diameter map generated from the FLIP recordings were used for evaluation of the luminal pressure and dynamic changes in the LES length, diameter, and axial movement (Fig. 1B). Details about the spatial-temporal diameter map plot from FLIP measurements can be found in papers by Fynne et $\mathrm{al}^{29}$ and Sorensen et al..$^{24}$

\section{Data processing}

In the spatio-temporal diameter map plot, a narrow zone along the LES was defined according to the diameter distribution along the distending bag (Fig. 1C). During the distension, the narrow

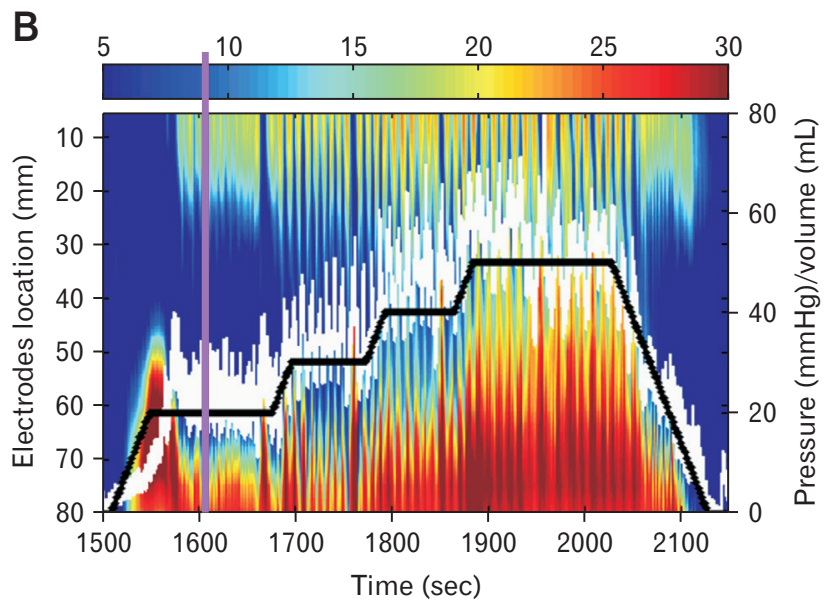

Figure 1. Functional luminal imaging probe (FLIP) assessment of the esophago-gastric junction. (A) Schematic diagram of the esophagogastric junction and the location of the FLIP during the test. (B) Example of spatial-temporal diameter map during the stepwise distension protocol. The map color from blue to red represents the diameter increase. The white line is the pressure change during the distension and the heavy black line is the volume change in the FLIP bag. The purple vertical line indicates the FLIP recording at time $=1610$ seconds. $(C)$ The 3D FLIP configuration at time 1610 seconds, ie, the purple line at (B), the map color from blue to red represents the diameter increase. The 5 red circle lines are the upper edge $\left(B_{\text {upper }}\right)$, proximal $\left(L_{\text {proximal }}\right)$, middle $\left(L_{\text {middle }}\right)$, distal $\left(L_{\text {distal }}\right)$ and lower edge $\left(B_{\text {lower }}\right)$ locations of lower esophageal sphincter (LES) narrow zone at the time point. 
zone was defined as the middle region with bag diameter $\leq 15$ $\mathrm{mm}$ for distension volumes $20 \mathrm{~mL}$ and $30 \mathrm{~mL}$, and $\leq 20 \mathrm{~mm}$ for distension volume $40 \mathrm{~mL}$. The narrow zone was distinguished by an upper edge towards the oral cavity $B_{\text {upper }}(t)$ and a lower edge towards the stomach $B_{\text {lower }}(t)$. The 2 boundaries were defined from the diameter map plot by using an edge detection algorithm. ${ }^{31}$

\section{Parameters for defining lower esophageal sphincter narrow zone motion}

During each distension volume, the narrow zone motion parameters were defined as: (1) length of the narrow zone, $L(t)$; (2) the narrow zone upper and lower boundary baseline, $\overline{B_{\text {upper }}(t)}$ and
$\overline{B_{\text {lower }}(t)}$; (3) the proximal, middle, and distal length of the narrow zone during the distension, $L_{\text {proximal }}(t), L_{\text {middle }}(t)$, and $L_{\text {distal }}(t)$; (4) the axial movement of the narrow zone, $L_{\text {movement }}(t)$; (5) the bag pressure change during the distension, $P(t)$; (6) the diameter change during distension in the distal, middle, and proximal parts of the narrow zone; and (7) the peristaltic velocity $v$.

Details on the definition of LES motion parameters are provided in the Appendix.

\section{Motion analysis}

The recorded pressure as well as the aforementioned motion parameters showed cyclic fluctuations at each distension volume
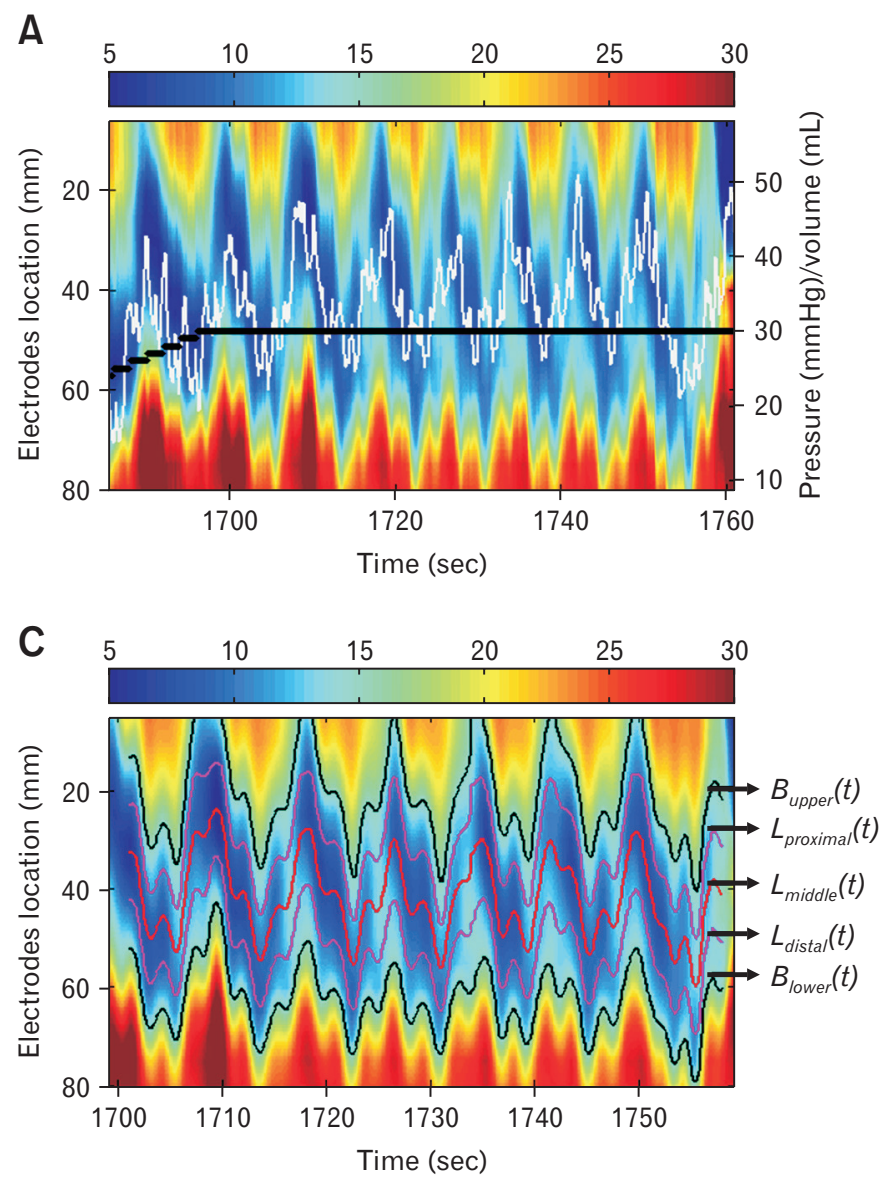
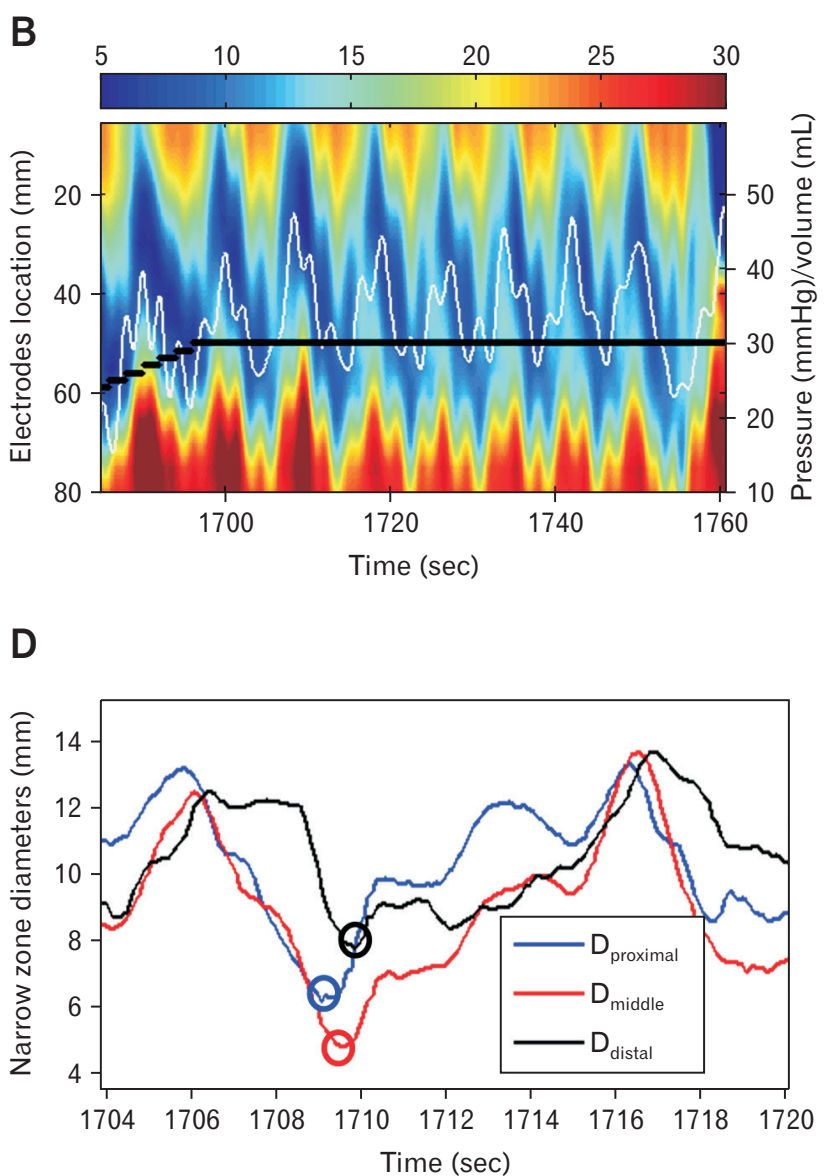

Figure 2. Example of pressure and diameter change before $(A)$ and after $(B)$ the low-pass filter. By using the low-pass filter, the signals with frequency $<30 \mathrm{cycle} / \mathrm{min}$ was passed and unchanged in (B), while the fluctuations due to heart action in (A) was successfully removed after the filter in (B). The white line is the pressure change during the distension and the heavy black line is the volume change in the functional luminal imaging probe bag. (C) The segmented lower esophageal sphincter (LES) narrow zone from the spatial-temporal diameter map shown in (B), where the upper edge $\left(B_{\text {upper }}(t)\right)$, lower edge $\left(B_{\text {lower }}(t)\right)$, proximal $\left(L_{\text {proximal }}(t)\right)$, middle $\left(L_{\text {middle }}(t)\right)$, and distal $\left(L_{\text {distal }}(t)\right)$ locations of the narrow zone were segmented during the distension. (D) Bag diameter during a cycle at the proximal, middle, and distal locations of the LES narrow zone. In the diameter cycle, the minimum diameter at the proximal narrow zone (blue circle) was reached at first and followed by the middle (red circle) and distal (black circle) of the narrow zone. 
(Fig. 1B). Fluctuations are due to distension, respiration, and heart rate. To remove artefacts induced by heart rate, all recorded data were filtered by using a low-pass filter where only signals with frequency $<30$ cycles/min were passed. The pressure at each disten- sion volume plateau was converted to the frequency domain using a fast Fourier transform (FFT) in order to distinguish the predominant factor for the phasic parameter change. A previous study by Shafik et $\mathrm{al}^{32}$ showed the esophageal motor activity induced by LES
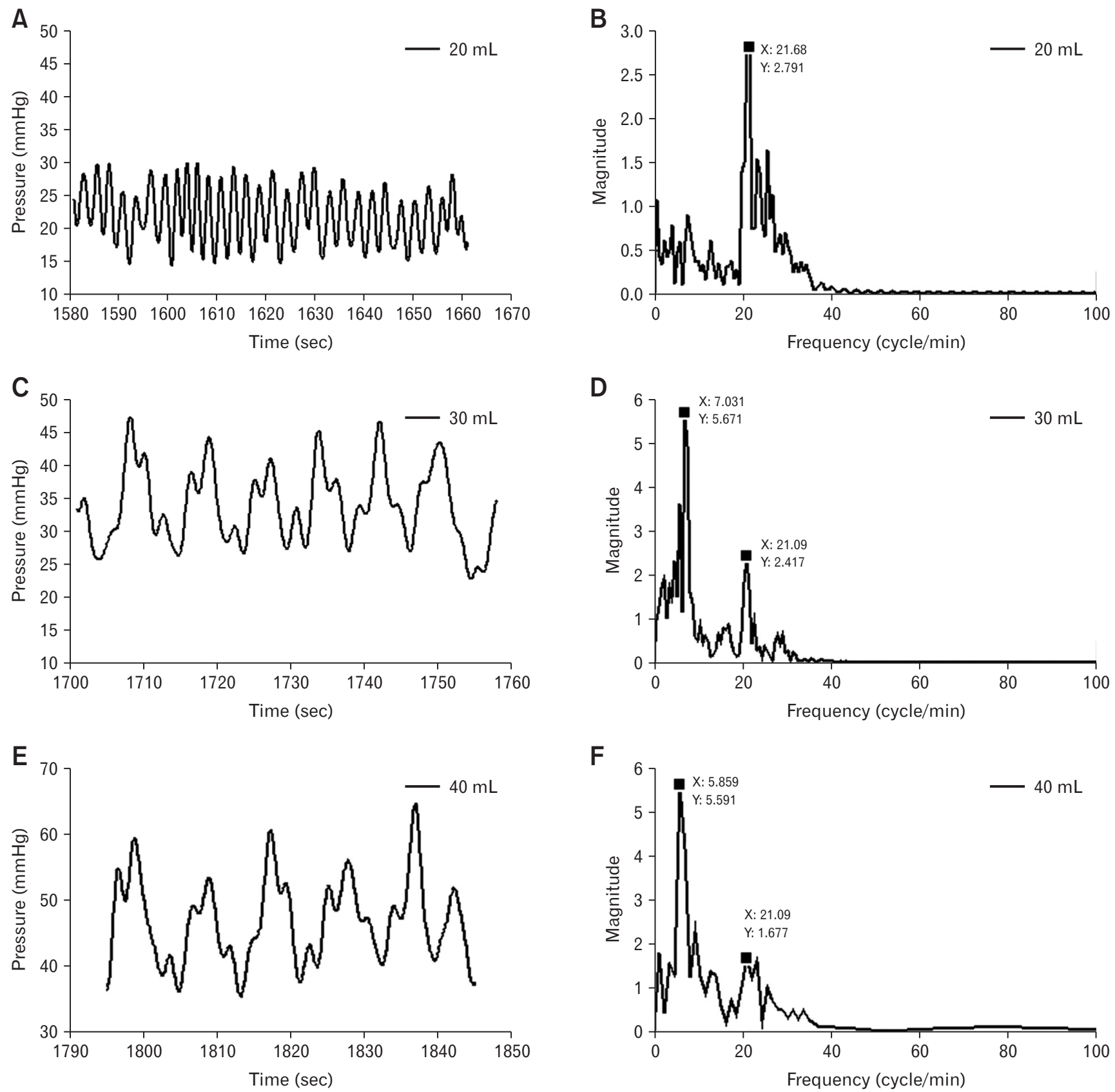

Figure 3. Example of the bag pressure change during distension volume $20 \mathrm{~mL}(\mathrm{~A}), 30 \mathrm{~mL}(\mathrm{C})$, and $40 \mathrm{~mL}$ (E), and the corresponding frequency response $(\mathrm{B}, \mathrm{D}, \mathrm{F})$. The frequency distribution in $(\mathrm{B})$ showed that pressure changes caused by respiration exhibit its primary frequency (the frequency with the highest amplitude) at 21.7 cycles/min. The frequency response in (D, F) showed the primary frequency for distension-induced contractions was 7.03 and 5.86 cycles/min. The pressure change caused by respiration had the secondary frequency (the frequency with the second highest amplitude) at 21.1 cycles/min (D, F). 
distension had frequencies in the range $8-10$ cycles/min. Therefore, in this study, if the fluctuation had a primary frequency $<12$ cycles/ $\mathrm{min}$, then the phasic change of the motion parameters were considered to be primarily induced by distension-evoked secondary peristaltic contractions. The typical respiratory rate for a healthy adult at rest is $12-20$ breaths per minute. Thus, if the primary frequency was between 12 and 25 cycles/min, the change of the parameters was considered to be caused by respiratory movements. For distensionevoked secondary peristaltic contractions, the peristaltic velocity $\mathrm{v}$ was calculated. All analyses were done using in-house-made Matlab subroutines (R2013; MathWorks, Natick, MA, USA).

\section{Statistical Methods}

The projected number of subjects, n, required to test a null hypothesis is given by the following equation:

$$
n>\left[\frac{\left[Z_{2 \alpha}+Z_{2 \beta}\right] \sigma}{\delta}\right]^{2}
$$

where $\alpha$ is the required probability of a Type I error, $\beta$ is the required probability of a Type II error, $\delta$ is the estimated difference between the means of 2 comparison groups, and $\sigma$ is the estimated standard deviation of the means in each of the 2 com- parison groups. We assume $2 \alpha<0.05\left(Z_{2 \alpha}=1.96\right)$ and $2 \beta<$ $0.10\left(Z_{2 \beta}=0.84\right)$. We assume the values of mean difference, $\delta$, and standard deviation, $\sigma$, from previous experience in our clinical laboratory and the sensitivity and specificity of current tests. The power analysis showed that 6 subjects would be sufficient to reach statistically valid conclusions. Data are presented as mean \pm standard error of the mean. A $t$ test was used for comparing the up-

Table. The Primary Frequency of Functional Luminal Imaging Probe Bag Pressure Fluctuations and the Averaged Upper Edge Location During Distensions for the 6 Subjects

\begin{tabular}{|c|c|c|c|c|c|c|}
\hline \multirow{2}{*}{$\begin{array}{c}\text { Subject } \\
\text { No }\end{array}$} & \multicolumn{2}{|c|}{$20 \mathrm{~mL}$} & \multicolumn{2}{|c|}{$30 \mathrm{~mL}$} & \multicolumn{2}{|c|}{$40 \mathrm{~mL}$} \\
\hline & $\begin{array}{c}\mathrm{PF} \\
\text { (cycle/min) }\end{array}$ & $\begin{array}{c}\mathrm{BU} \\
(\mathrm{mm})\end{array}$ & $\begin{array}{c}\mathrm{PF} \\
\text { (cycle/min) }\end{array}$ & $\begin{array}{c}\mathrm{BU} \\
(\mathrm{mm})\end{array}$ & $\begin{array}{c}\mathrm{PF} \\
\text { (cycle/min) }\end{array}$ & $\begin{array}{c}\mathrm{BU} \\
(\mathrm{mm})\end{array}$ \\
\hline 1 & 21.1 & 20.1 & 7.0 & 20.8 & 5.9 & 14.0 \\
\hline 2 & 18.8 & 10.4 & 17.6 & 8.0 & 16.4 & 7.6 \\
\hline 3 & 16.4 & 7.5 & 7.0 & 22.8 & 4.69 & 36.0 \\
\hline 4 & 12.9 & 30.9 & 14.1 & 33.7 & 14.1 & 20.7 \\
\hline 5 & 21.1 & 15.5 & 18.8 & 8.6 & 7.6 & 20.4 \\
\hline 6 & 11.7 & 20.1 & 17.5 & 21.0 & 19.1 & 23.4 \\
\hline
\end{tabular}

$\mathrm{PF}$, primary frequency; $\mathrm{BU}$, the averaged upper edge, ie, the upper boundary baseline $\left(\overline{B_{\text {upper }}(t)}\right)$ for each distension.
A

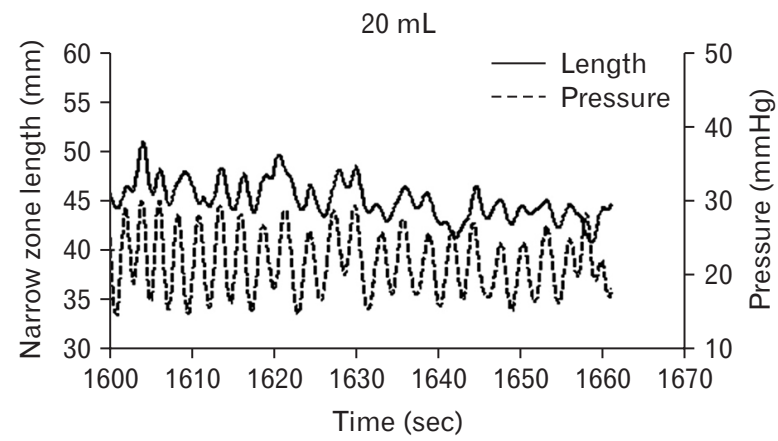

C

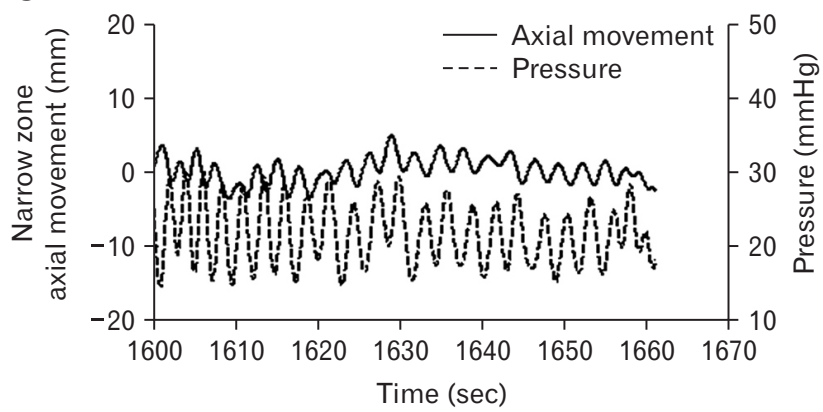

B

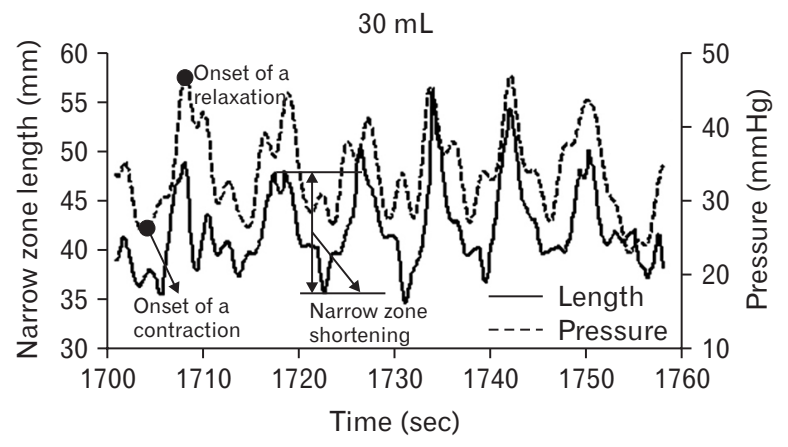

D

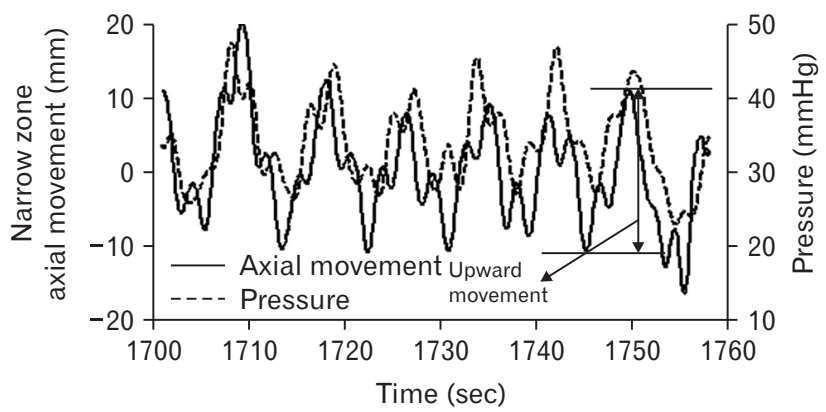

Figure 4. Example of the bag pressure and the synchronous length change (A, B) and the axial movement (C, D) of the lower esophageal sphincter (LES) narrow zone at distension volumes $20 \mathrm{~mL}(\mathrm{~A}, \mathrm{C})$ and $30 \mathrm{~mL}(\mathrm{~B}, \mathrm{D})$. The definitions of the LES narrow zone shortening and the upward axial movement of the narrow zone are illustrated in panel $\mathrm{B}$ and $\mathrm{D}$. 
per boundary baselines between the distensions with and without secondary peristaltic contractions and parameters between volume $30 \mathrm{~mL}$ and $40 \mathrm{~mL}$. One-way analysis of variance (ANOVA) was used for comparing parameters to the distension volumes and twoway ANOVA was used to distinguish the distension volume and the LES narrow zone motion parameters difference with factor $1=$ distension volume and factor $2=$ respiration vs peristaltic contraction. Statistical calculations were done using SigmaPlot 11.0 (Systat Software Inc, San Jose, CA, USA). Tukey test and Dunn's method were used as the post hoc test for parametric and non-parametric distribution data analysis. $P<0.05$ was considered statistically significant.

\section{Results}

The LES was almost fully distended at the distension volume of $50 \mathrm{~mL}$, which made it difficult to define the narrow zone. Hence, only data at distension volumes 20,30 , and $40 \mathrm{~mL}$ were analyzed.
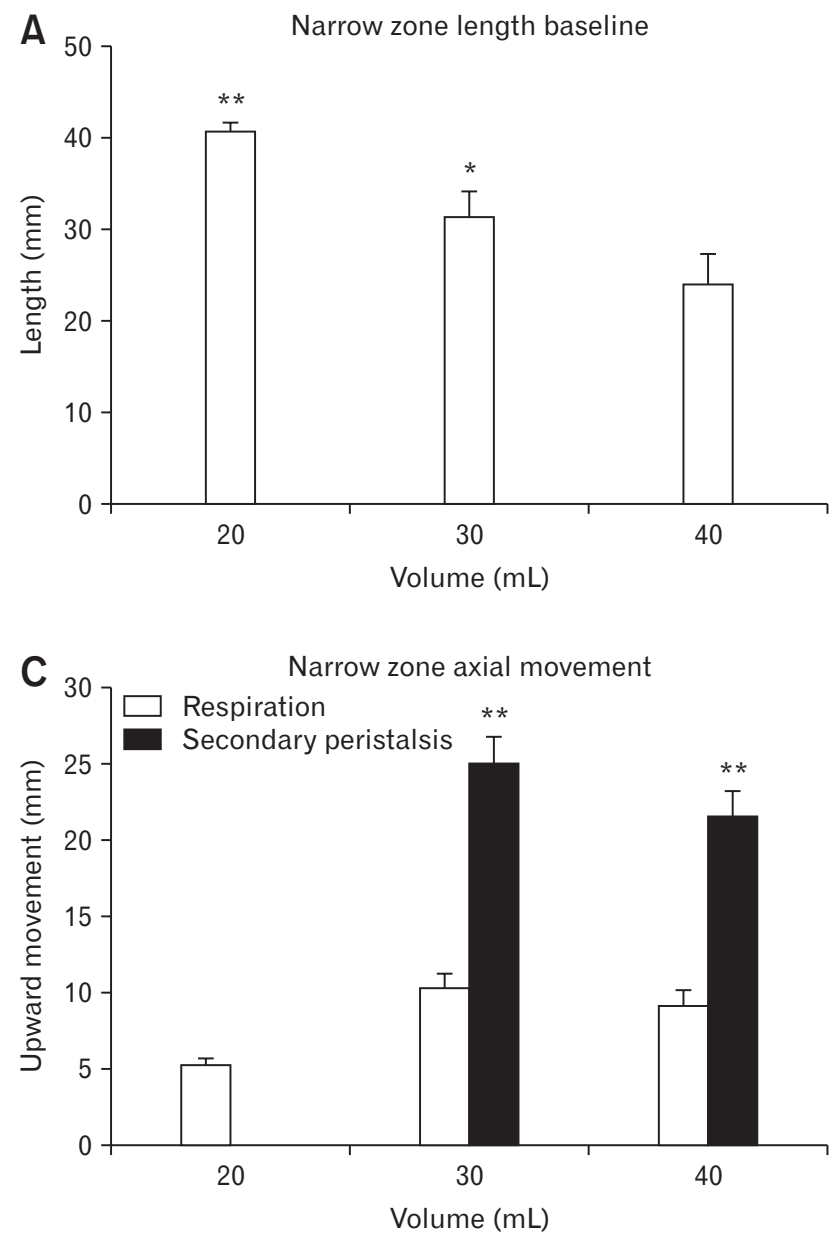

Visual inspection of pressure and diameter change before and after the low-pass filtering showed that pressure fluctuations induced by heart rate were successfully removed by the filter. Signals with frequency $<30$ cycles/min passed unchanged (Fig. 2A and 2B). All results below are based on filtered FLIP recordings.

\section{Lower Esophageal Sphincter Narrow Zone Segmentation}

An example of the segmented upper edge, lower edge, and the proximal, middle, and distal location of the LES narrow zone is shown in Figure 2C.

\section{Lower Esophageal Sphincter Narrow Zone Motion During the Distensions}

The pressure changes at distension volumes 20, 30, and $40 \mathrm{~mL}$ showed pressure fluctuations due to respiration with a frequency of $16.8 \pm 0.8$ cycles/min (Fig. 3A and 3B, Table), and pressure fluctuations due to distension-induced secondary peristaltic con-

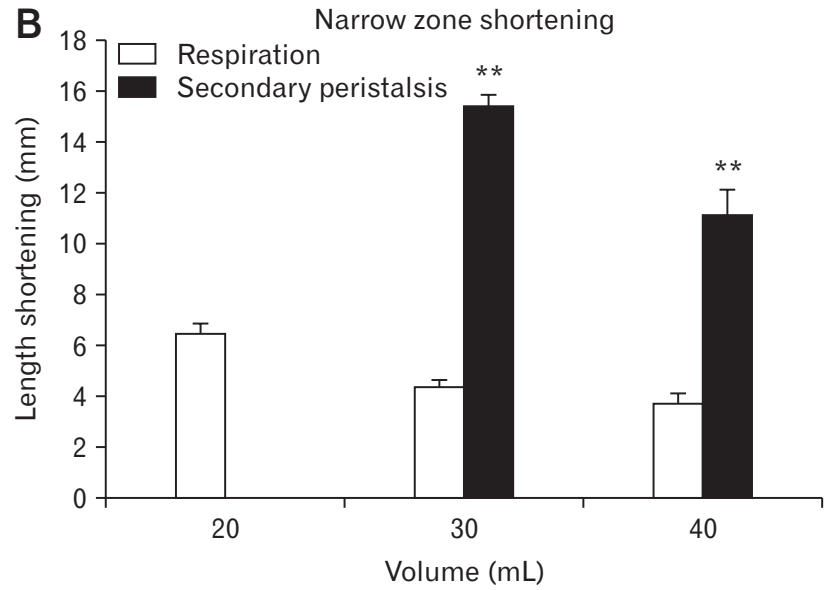

Figure 5. The longitudinal motion of the lower esophageal sphincter (LES) narrow zone during distension. (A) The narrow zone length baseline, ie, the averaged narrow zone length. (B) The narrow zone length shortening. (C) The narrow zone axial movement. ${ }^{*} P<0.05$ and ${ }^{*} P<$ 0.001 . 
tractions with a frequency of $6.4 \pm 0.5$ cycles/min (Fig. 3C-F, Table). Pressure fluctuations induced by secondary peristalsis were superimposed on those induced by respiration (Fig. 3C-F). For both types of induced pressure fluctuations, the frequencies were independent of the distension volume ( $\mathrm{F}<0.74, P>0.45$; Table). The distension triggered secondary peristalsis at volume $30 \mathrm{or} / \mathrm{and}$ $40 \mathrm{~mL}$, presented as a lower pressure primary frequency and propagations of the peristaltic diameter waves along the probe (Fig. 2D). However, in 3 of the 6 subjects, there was no peristaltic response recorded during the distensions (Table). The upper boundary baseline at distension volumes $30 \mathrm{~mL}$ and $40 \mathrm{~mL}$ tended to be larger for distensions triggering peristaltic contraction than those without secondary peristaltic contractions $(22.8 \pm 3.6 \mathrm{~mm}$ vs $17.6 \pm 3.0$ $\mathrm{mm}, P=0.173)$.
A
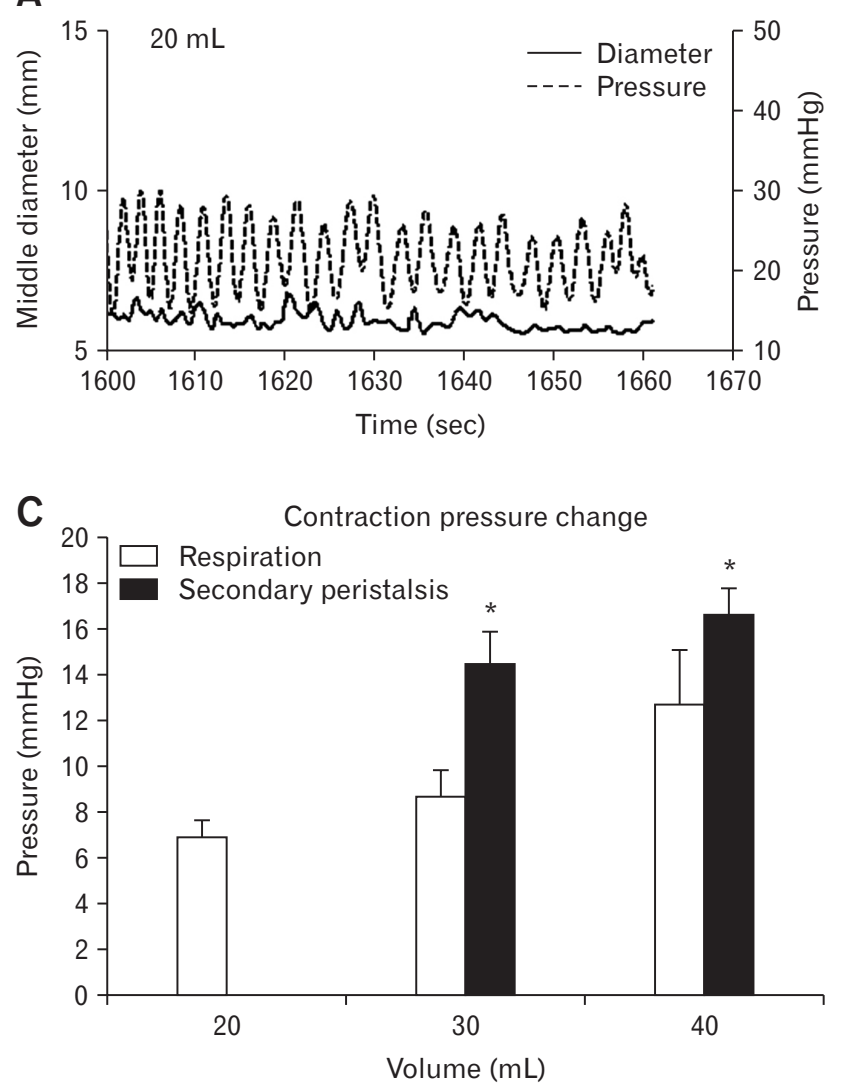

\section{Longitudinal Motion of the Lower Esophageal Sphincter Narrow Zone}

An example of narrow zone length change and the axial movement at distension volume $20 \mathrm{~mL}$ and $30 \mathrm{~mL}$ are shown in Figure 4, representing the LES longitudinal motion and the synchronous pressure change induced by respiration and secondary peristalsis. During secondary peristalsis, the narrow zone shortened after the onset of the pressure relaxation whereas LES axial movement started at the onset of the pressure contraction (Fig. 4). During the distension, the narrow zone length shortened as function of the volume, from $40.6 \pm 1.0 \mathrm{~mm}$ at volume $20 \mathrm{~mL}$ to $24.0 \pm 3.2 \mathrm{~mm}$ at volume $40 \mathrm{~mL}$ ( $\mathrm{F}=36.1, P<0.001$; Fig. $5 \mathrm{~A})$. Compared to the fluctuation induced by respiration, secondary peristalsis induced more pronounced LES narrow zone shortening and axial movement at all distension volumes ( $\mathrm{F}>5.4, P<0.007$; Fig. 5B and $5 \mathrm{C})$. For the longitudinal motion induced by secondary peristalsis,
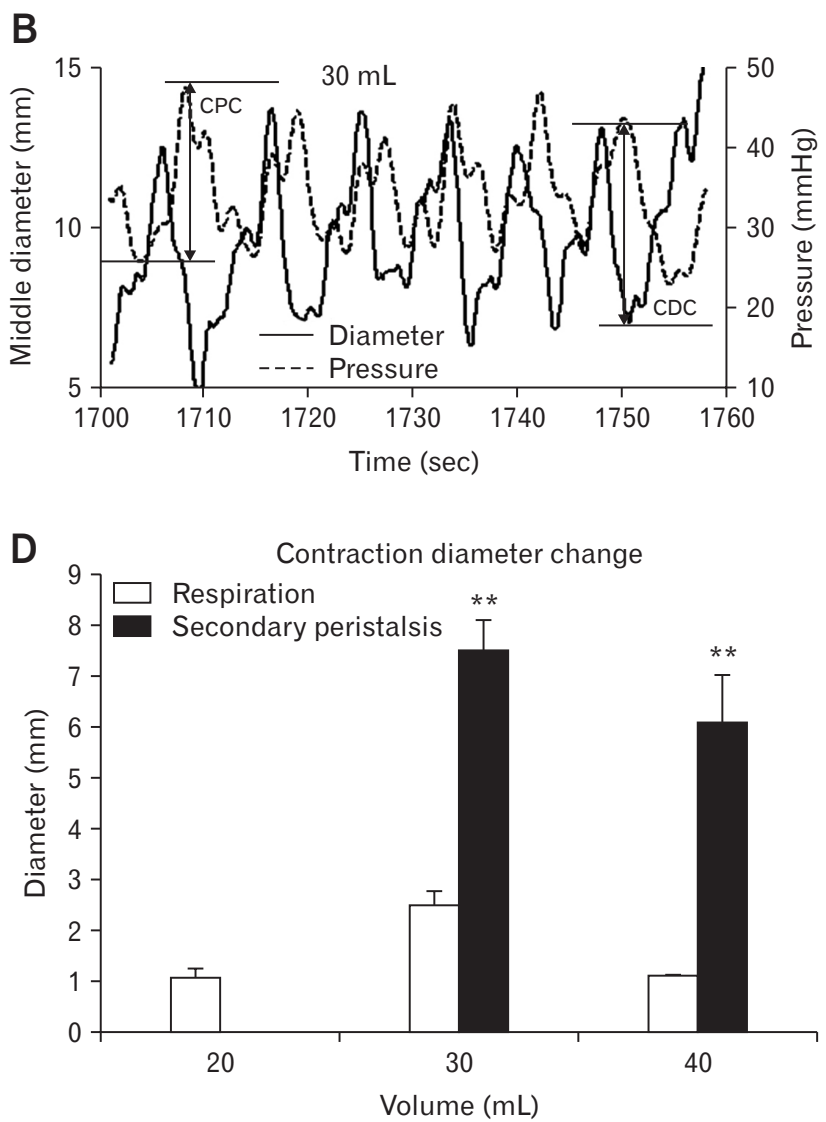

Figure 6. The circumferential motion of the lower esophageal sphincter (LES) narrow zone during distensions. Example of the bag pressure and the synchronous middle diameter change of the LES narrow zone at distension volume $20 \mathrm{~mL}$ (A) and $30 \mathrm{~mL}$ (B). The definitions of the contraction pressure change $(\mathrm{CPC})$ and the contraction diameter change $(\mathrm{CDC})$ are illustrated in panel $\mathrm{B}$. The contraction pressure change during the distensions (C). The contraction diameter change during the distensions (D). ${ }^{*} P<0.01$ and ${ }^{* *} P<0.001$. 
the distension volume did not influence LES narrow zone axial movement $(P=0.573)$ whereas the narrow zone shortened most at distension volume $30 \mathrm{~mL}(P=0.004)$.

\section{Circumferential Motion of the Lower Esophageal Sphincter Narrow Zone}

At distension volumes 20,30 , and $40 \mathrm{~mL}$, the bag pressure increased from $16.3 \pm 0.5 \mathrm{mmHg}$ to $27.4 \pm 1.2 \mathrm{mmHg}$ and $40.2 \pm 1.5 \mathrm{mmHg}(\mathrm{F}=81.4, P<0.001)$. At the same time, the narrow zone was dilated with the middle diameter increasing from $7.2 \pm 0.5 \mathrm{~mm}$ to $9.8 \pm 1.1 \mathrm{~mm}$ and $14.2 \pm 0.8 \mathrm{~mm}$ ( $\mathrm{F}=85.8, P<0.001)$. Examples of bag pressure and diameter changes at distension volumes $20 \mathrm{~mL}$ and $30 \mathrm{~mL}$ are shown in Figure 6A and 6B. The amplitudes of contraction pressure and contraction diameter changes induced by secondary peristalsis were significantly higher than those induced by respiration (q $>4.2, P<0.003$; Fig. $6 \mathrm{C}$ and $6 \mathrm{D})$ ). The contraction pressure change increased with the distension volume $(\mathrm{F}=$ 5.5, $P=0.005$; Fig. $6 \mathrm{C})$. The contraction diameter change was largest at volume $30 \mathrm{~mL}$ (q $=3.46, P=0.043$ ). During the secondary peristalsis, the middle diameter began to decrease about 2 seconds after the onset of the pressure increase (Fig. 6B). The propagation velocity of the secondary peristalsis at distension 30 $\mathrm{mL}$ was higher than that at volume $40 \mathrm{~mL}(21.8 \pm 3.5 \mathrm{~mm} / \mathrm{sec}$ vs $12.6 \pm 1.8 \mathrm{~mm} / \mathrm{sec}, P=0.034)$.

\section{Associations Between the Pressure Change and Lower Esophageal Sphincter Narrow Zone Length}

The length change of the narrow zone was associated with pressure increase. For each subject, the relationship between the FLIP bag pressure and the narrow zone length in all distension volumes could be described by the linear regression model Narrow zone length $=a^{*}$ pressure $+b$, where a represents the LES narrow zone shortening to the pressure increase and $b$ represents the initial LES narrow zone length, ie, the length at zero pressure. The constants a and $b$ from all 6 subjects were $-0.39 \pm 0.12 \mathrm{~mm} / \mathrm{mmHg}$ and $42.9 \pm$ $3.6 \mathrm{~mm}$.

\section{Discussion}

Respiration as well as bag distension induced dynamic pressure and geometric changes of the LES. Based on FLIP measurements, the dynamic changes of the LES can be distinguished and described by circumferential and longitudinal motion parameters. This is the first study where the longitudinal motion of LES, in- duced by respiration and secondary peristalsis, was analyzed by using FLIP technology. Such data cannot be obtained by non-distension technologies like HRM. For the same reason such techniques cannot be used to validate the data presented. Major findings in this study are: (1) both respiration and secondary peristalsis induced synchronous cyclic longitudinal and circumferential motions in the LES region; (2) compared to fluctuations caused by respiration, secondary peristalsis induced significantly more shortening, axial movement, contraction pressure change, and contraction diameter change of the narrow zone; and (3) the LES narrow zone length change was associated with the luminal LES pressure.

Compared with existing methods for LES longitudinal motion measurement, this study moved a step further by providing a combined circumferential and longitudinal motility analysis on the basis of FLIP measurements. We are capable of quantitatively evaluating LES shortening, LES axial movement as well as the synchronized LES diameter change induced by respiration and peristaltic contraction using a single technology.

Similar to findings by Dodds et $\mathrm{al}^{10}$ in the feline esophagus, our study showed diaphragmatic contractions moving and shortening the narrow zone in rhythm with the respiration-induced LES pressure fluctuations at resting and low volume distensions $(20 \mathrm{~mL})$. And the pressure increase caused by inspiration depended on the distension volume, the higher volume, the higher pressure. The maximum LES pressure increase induced by inspiration was 12.7 $\pm 2.4 \mathrm{mmHg}$ in this study. The magnitude was much less than that reported by Welch and $\mathrm{Gray}^{20}(>60 \mathrm{mmHg}$ ) and Mittal et $\mathrm{al}^{33}$ (> $40 \mathrm{mmHg}$ ), where the maximum LES pressure increase were calculated on the basis of manometric measurement at the maximum inspiratory depth. In the present study the bag pressures were obtained at a normal inspiratory force, which may explain the difference between the studies. LES axial movement induced by respiration was $5.3-10.3 \mathrm{~mm}$ in this study, which is in agreement with data using pull-through technology $(2.0-12.0 \mathrm{~mm}) .^{20}$

Compared to LES motions associated with respiration, both circumferential and longitudinal motions caused by distensioninduced secondary peristaltic contractions were more dramatic. The maximum axial movement and the maximum shortening of the LES occurred at distension volume $30 \mathrm{~mL}$ (Fig. 5). The magnitude of the axial movement $(25.0 \mathrm{~mm})$ and the LES shortening ratio $(15.4 \mathrm{~mm} / 42.9 \mathrm{~mm}$ at the initial state $=35.9 \%)$ in this study are consistent with a previous clips study on secondary peristalsisinduced longitudinal shortening and movement of the most distal part of the esophagus, ${ }^{14}$ indicating the reliability and validity of the present study. Previous studies on esophageal propulsive force in 
response to fixed balloon distension showed that the distensioninduced propulsive force was not by itself a peristaltic wave, as it may persist locally for long periods rather than progressing down the esophagus. ${ }^{34}$ However, in our previous study using soft bag distension in the esophagus, the esophageal contractions evoked by distensions propagated along the esophagus and passed the bag even at the highest distension pressure. ${ }^{35}$ Furthermore, according to Shafik et al, ${ }^{32}$ LES dilation can initiate esophageal contractions with increased esophageal pressure and excess electromyographic activity. The measurement in the present study confirmed these views. When the distension volume was $\geq 30 \mathrm{~mL}$, the bag pressure increased dramatically with a lower pressure frequency, and the contraction propagated abnormally along the bag (Fig. 2D). It has been demonstrated in the human esophagus that the secondary peristaltic response rate to bolus and balloon distension is volume dependent, ie, higher volume distension was more effective in triggering secondary peristalsis. ${ }^{36}$ This could be an explanation for the secondary peristalsis that occurred at volume $\geq 30 \mathrm{~mL}$ in this study. However, it is worthwhile to note that the peristaltic response was not triggered in 3 of 6 subjects. Two of these subjects had a lower upper edge than those with peristaltic contractions, and the third had a significant FLIP probe upward movement during distensions (data not shown). The lower upper edge means that a smaller part of the bag at the proximal end was located in the tubular esophagus, ie, above the phrenic ampulla. Simultaneous fluoroscopic and manometric studies of the EGJ by Lin et $\mathrm{al}^{37}$ suggested that the phrenic ampulla is physiologically distinct from the esophagus. Hence, the secondary peristalsis likely depends on how much of the bag is located in the mid esophagus. The present study clearly shows that FLIP distension induces secondary peristalsis which is consistent with many previous FLIP studies. Most likely, the secondary peristalsis induced by the bag part located in the lower esophageal body.

Despite demonstration of LES circumferential and longitudinal motions induced by FLIP distension, several methodological limitations should be considered in interpreting these findings. The spatio-temporal diameter maps were generated based on linear interpolation of the FLIP measurement. Since the cross-sectional area measurements are made at $5 \mathrm{~mm}$ intervals, the defined LES narrow zone could not be $100 \%$ accurate. Furthermore, the definition of upper and lower edges of the narrow zone requires correct placement of the bag in the narrow zone. About half of the studies we considered for analysis fell for the criteria that the whole LES region had to be visible in the recordings at all times. However, according to the power analysis, 6 samples were sufficient to reach statistically valid conclusions. One methodological issue relates to whether it is physiologically relevant to discuss esophageal lengthening. However, the length of the esophagus is balanced by the passive mechanical properties as well as the active muscle properties and the degree of stretch imposed by other organs in vivo. If the esophagus becomes shorter during contraction, it implies that it will lengthen during relaxation. It has been demonstrated in many studies that the esophageal body is capable of generating tonic contractions. Hence at physiological conditions it is likely capable of changing its length to shorten or elongate.

In conclusion, studies conducted during the last 2 decades have made it clear that esophageal longitudinal muscles play an important physiological and pathophysiological role for motility disorders and for the etiology of dysphagia and esophageal pain. ${ }^{2,19,38}$ Compared with technologies for measurement of circumferential muscle activity in the esophagus and EGJ, a major problem in studying the longitudinal muscle activity of the esophagus is that we still lack behind in technology for monitoring longitudinal movements of the LES over a prolonged period of time. By analyzing FLIP measurements in the present study, the dynamic changes of the LES could be distinguished and quantitatively determined by circumferential and longitudinal motion parameters. The present FLIP study with advanced analysis may shed more light on esophageal disorders associated with abnormal longitudinal muscle activity in the future.

Financial support: This study was supported by grants from the Karen Elise Jensen Foundation (Project number: 903959).

Conflicts of interest: Hans Gregersen and Barry P McMahon invented the FLIP technology and hold minority shares in Crospon Ltd. The other authors declare no conflict of interests regarding the publication of this article.

Author contributions: Study concept and design and critical revision of manuscript: Donghua Liao, Christian Lottrup, Lotte Fynne, Barry P McMahon, Klaus Krogh, Asbjørn M Drewes, Jingbo Zhao, and Hans Gregersen; data collection: Christian Lottrup and Lotte Fynne; data analysis: Donghua Liao, Hans Gregersen, Christian Lottrup, Lotte Fynne, and Jingbo Zhao; and manuscript preparation: Donghua Liao, Hans Gregersen, and Christian Lottrup.

\section{References}

1. Mittal RK, Balaban DH. The esophagogastric junction. N Engl J Med 1997;336:924-932. 
2. Mittal RK, Hong SJ, Bhargava V. Longitudinal muscle dysfunction in achalasia esophagus and its relevance. J Neurogastroenterol Motil 2013;19:126-136.

3. Sugarbaker DJ, Rattan S, Goyal RK. Mechanical and electrical activity of esophageal smooth muscle during peristalsis. Am J Physiol 1984;246:G145-G150.

4. Gregersen H, Drewes AM, McMahon BP, Liao D. Balloon-distension studies in the gastrointestinal tract: current role. Dig Dis 2006;24:286296.

5. Lottrup C, McMahon BP, Ejstrud P, Ostapiuk MA, Funch-Jensen P, Drewes AM. Esophagogastric junction distensibility in hiatus hernia. Dis Esophagus 2016;29: 463-471.

6. McMahon BP, Frokjaer JB, Kunwald P, et al. The functional lumen imaging probe (FLIP) for evaluation of the esophagogastric junction. Am J Physiol Gastrointest Liver Physiol 2007;292:G377-G384.

7. Mittal RK, Liu J, Puckett JL, et al. Sensory and motor function of the esophagus: lessons from ultrasound imaging. Gastroenterology 2005;128:487-497.

8. Mittal RK, Karstens A, Leslie E, Babaei A, Bhargava V. Ambulatory high-resolution manometry, lower esophageal sphincter lift and transient lower esophageal sphincter relaxation. Neurogastroenterol Motil 2012;24:40-46, e2.

9. Stein HJ, Liebermann-Meffert D, DeMeester TR, Siewert JR. Threedimensional pressure image and muscular structure of the human lower esophageal sphincter. Surgery 1995;117:692-698.

10. Dodds WJ, Stewart ET, Hodges D, Zboralske FF. Movement of the feline esophagus associated with respiration and peristalsis. An evaluation using tantalum markers. J Clin Invest 1973;52:1-13.

11. Edmundowicz SA, Clouse RE. Shortening of the esophagus in response to swallowing. Am J Physiol 1991;260:G512-G516.

12. Pandolfino JE, Zhang QG, Ghosh SK, Han A, Boniquit C, Kahrilas PJ. Transient lower esophageal sphincter relaxations and reflux: mechanistic analysis using concurrent fluoroscopy and high-resolution manometry. Gastroenterology 2006;131:1725-1733.

13. Pouderoux P, Lin S, Kahrilas PJ. Timing, propagation, coordination, and effect of esophageal shortening during peristalsis. Gastroenterology 1997;112:1147-1154.

14. Shi G, Pandolfino JE, Joehl RJ, Brasseur JG, Kahrilas PJ. Distinct patterns of oesophageal shortening during primary peristalsis, secondary peristalsis and transient lower oesophageal sphincter relaxation. Neurogastroenterol Motil 2002;14:505-512.

15. Miller LS, Liu JB, Colizzo FP, et al. Correlation of high-frequency esophageal ultrasonography and manometry in the study of esophageal motility. Gastroenterology 1995;109:832-837.

16. Mittal RK, Padda B, Bhalla V, Bhargava V, Liu J. Synchrony between circular and longitudinal muscle contractions during peristalsis in normal subjects. Am J Physiol Gastrointest Liver Physiol 2006;290:G431G438.

17. Nicosia MA, Brasseur JG, Liu JB, Miller LS. Local longitudinal muscle shortening of the human esophagus from high-frequency ultrasonography. Am J Physiol Gastrointest Liver Physiol 2001;281:G1022-G1033.

18. Pehlivanov N, Liu J, Kassab GS, Puckett JL, Mittal RK. Relation- ship between esophageal muscle thickness and intraluminal pressure: an ultrasonographic study. Am J Physiol Gastrointest Liver Physiol 2001;280:G1093-G1098.

19. Dai Q, Korimilli A, Thangada VK, et al. Muscle shortening along the normal esophagus during swallowing. Dig Dis Sci 2006;51:105-109.

20. Welch RW, Gray JE. Influence of respiration on recordings of lower esophageal sphincter pressure in humans. Gastroenterology 1982;83:590594.

21. Babaei A, Bhargava V, Korsapati H, Zheng WH, Mittal RK. A unique longitudinal muscle contraction pattern associated with transient lower esophageal sphincter relaxation. Gastroenterology 2008;134:1322-1331.

22. Lee YY, Whiting JG, Robertson EV, Derakhshan MH, Smith D, McColl KE. Measuring movement and location of the gastroesophageal junction: research and clinical implications. Scand J Gastroenterol 2013;48:401-411.

23. McMahon BP, Frøkjaer JB, Liao D, Kunwald P, Drewes AM, Gregersen $\mathrm{H}$. A new technique for evaluating sphincter function in visceral organs: application of the functional lumen imaging probe (FLIP) for the evaluation of the oesophago-gastric junction. Physiol Meas 2005;26:823836.

24. Sorensen G, Liao D, Lundby L, et al. Distensibility of the anal canal in patients with idiopathic fecal incontinence: a study with the Functional Lumen Imaging Probe. Neurogastroenterol Motil 2014;26:255-263.

25. Lottrup C, Gregersen H, Liao D, et al. Functional lumen imaging of the gastrointestinal tract. J Gastroenterol 2015;50:1005-1016.

26. Carlson DA, Lin Z, Kahrilas PJ, et al. The Functional lumen imaging probe detects esophageal contractility not observed with manometry in patients with achalasia. Gastroenterology 2015;149:1742-1751.

27. Teitelbaum EN, Sternbach JM, El KR, et al. The effect of incremental distal gastric myotomy lengths on EGJ distensibility during POEM for achalasia. Surg Endosc 2016;30:745-750.

28. Carlson DA, Kahrilas PJ, Lin Z, et al. Evaluation of esophageal motility utilizing the runctional lumen imaging probe. Am J Gastroenterol 2016;111:1726-1735.

29. Fynne L, Liao D, Aksglaede K, et al. Esophagogastric junction in systemic sclerosis: A study with the functional lumen imaging probe. Neurogastroenterol Motil Published Online First: 3 May 2017. doi: 10.1111/ nmo.13073.

30. Fynne L, Luft F, Gregersen H, et al. Distensibility of the anal canal in patients with systemic sclerosis: a study with the functional lumen imaging probe. Colorectal Dis 2013;15:e40-e47.

31. Liao D, Zhao J, Gregersen H. Regional surface geometry of the rat stomach based on three-dimensional curvature analysis. Phys Med Biol 2005;50:231-246.

32. Shafik A, Shafik I, El-Sibai O, Mostafa R. Effect of lower esophageal sphincter distension and acidification on esophageal pressure and electromyographic activity: the identification of the "sphinctero-esophageal excitatory reflex”. Ann Thorac Surg 2005;79:1126-1131, discussion 1131.

33. Mittal RK, Rochester DF, McCallum RW. Effect of the diaphragmatic contraction on lower oesophageal sphincter pressure in man. Gut 1987;28:1564-1568.

34. Winship DH, Zboralske FF. The esophageal propulsive force: esopha- 
geal response to acute obstruction. J Clin Invest 1967;46:1391-1401.

35. Liao D, Villadsen GE, Gregersen H. Distension-evoked motility analysis in human esophagus. Neurogastroenterol Motil 2013;25:407, e296e297.

36. Schoeman MN, Holloway RH. Stimulation and characteristics of secondary oesophageal peristalsis in normal subjects. Gut 1994;35:152-158.
37. Lin S, Brasseur JG, Pouderoux P, Kahrilas PJ. The phrenic ampulla: distal esophagus or potential hiatal hernia? Am J Physiol 1995;268:G320G327.

38. Paterson WG. Studies on opossum esophageal longitudinal muscle function. Can J Physiol Pharmacol 1997;75:65-73. 